\title{
Review
}

\section{Learning in a Simple Motor System}

\author{
Dianne M. Broussard ${ }^{1}$ and Charles D. Kassardjian \\ Division of Cellular and Molecular Biology, Toronto Western Research Institute, University of Toronto, Toronto, \\ Ontario M5T 2S8, Canada
}

\begin{abstract}
Motor learning is a very basic, essential form of learning that appears to share common mechanisms across different motor systems. We evaluate and compare a few conceptual models for learning in a relatively simple neural system, the vestibulo-ocular reflex (VOR) of vertebrates. We also compare the different animal models that have been used to study the VOR. In the VOR, a sensory signal from the semicircular canals is transformed into a motor signal that moves the eyes. The VOR can modify the transformation under the guidance of vision. The changes are persistent and share some characteristics with other types of associative learning. The cerebellar cortex is directly linked to the VOR reflex circuitry in a partnership that is present in all vertebrates, and which is necessary for motor learning. Early theories of Marr, Albus, and Ito, in which motor memories are stored solely in the cerebellar cortex, have not explained the bulk of the experimental data. Many studies appear to indicate a site of learning in the vestibular nuclei, and the most successful models have incorporated long-term memory storage in both the cerebellar cortex and the brainstem. Plausible cellular mechanisms for learning have been identified in both structures. We propose that short-term motor memory is initially stored in the cerebellar cortex, and that during consolidation of the motor memory the locus of storage shifts to include a brainstem site. We present experimental results that support our hypothesis.
\end{abstract}

In many situations, inaccurate movements are worse than useless. If the movement is slow, errors can be corrected online by visual or other sensory feedback. However, rapid movements must be intrinsically accurate. This could be accomplished by hard-wiring an accurate movement, which has the obvious disadvantage that the movement cannot be readjusted for changes in downstream motor centers or (in the case of a reflex) for changes in sensory sensitivity. A better solution is to calibrate the movement by trial and error, that is, by motor learning. For our purposes, motor learning is defined as the process by which we acquire precise, coordinated movements, like those that are required in order to run, serve a tennis ball, and type accurately. Because accurate movements are necessary whenever predators must be avoided or prey captured, we can speculate that motor learning may have been one of the earliest types of associative learning to evolve. It has been demonstrated in the ventral nerve cord of insects (Horridge 1962) and is probably universal in freely-moving animals. Furthermore, because its substrate can be relatively simple and well understood, there is a real possibility of understanding motor learning both on the molecular level and on the level of brain circuitry.

Motor learning is classified as procedural learning. Errors are monitored and used to guide adaptive changes without conscious awareness. Instead of learning a concept, the subject learns an accurate and appropriate motor response. Distinctions have also been made between short- and long-term motor memory (separated by the process of consolidation). In the laboratory, consistent with accepted classical conditioning paradigms, motor learning involves pairing of conditioned and unconditioned stimuli, with the success of learning depending on their temporal relationship. However, in the real world, operant strategies may be used, in which a movement is made and sensory feedback is used to evaluate its accuracy.

The vestibulo-ocular reflex (VOR) is a relatively simple mo-

1 Corresponding author.

E-MAIL dianne@uhnres.utoronto.ca; FAX (416) 603-5745.

Article and publication are at http://www.learnmem.org/cgi/doi/10.1101/ Im.65804. tor control system that offers the opportunity to understand mammalian motor learning on the cellular, and potentially the molecular, level. Because the VOR pathway is relatively short (see Fig. 1A), the number of possible mechanisms that must be considered is comparatively small. Eye movements can be treated as pure rotations, which makes them easy to describe quantitatively. Because eye movements are kinematically simpler than limb movements, it is easier to establish a close correspondence between their parameters and neurophysiological measurements than it is for any other type of movement. The oculomotor system can be mathematically modeled as a simple linear control system (for review, see Robinson 1981), and the model is fairly accurate within a moderate range of stimulus parameters. The modeling approach has been used successfully to solve important problems in motor learning (for example, see Lisberger 1994).

In this review, we will deal first with general mechanisms for VOR motor learning and then with the specific role of the cerebellum. Issues that have attracted interest recently, such as generalization of motor learning to different stimuli, possible cellular mechanisms for learning, and consolidation of motor memory, will be mentioned. Finally, we will evaluate the relative advantages and disadvantages of some of the animal models that have been used to study VOR motor learning.

\section{Why is Motor Learning Necessary in the VOR?}

The VOR generates eye movements that stabilize gaze by compensating for head movements. This presumably prevents the visual image from blurring due to "slip" with respect to photoreceptors, so that while walking down the street (for example) it is possible to read signs and recognize faces (J.C. 1952). The VOR can perform accurately at velocities of up to $360^{\circ} / \mathrm{sec}$ (Paige 1983) and frequencies as high as $25 \mathrm{~Hz}$ (Huterer and Cullen 2002). Because visual feedback is too slow to be useful in the normal operation of the VOR, the transformation between the vestibular sensory input and the motor output of the reflex must be calibrated precisely. In the VOR system, changes in performance can be compared directly with changes in the responses of premotor neurons during the behavior. Motor learning in the VOR is also 


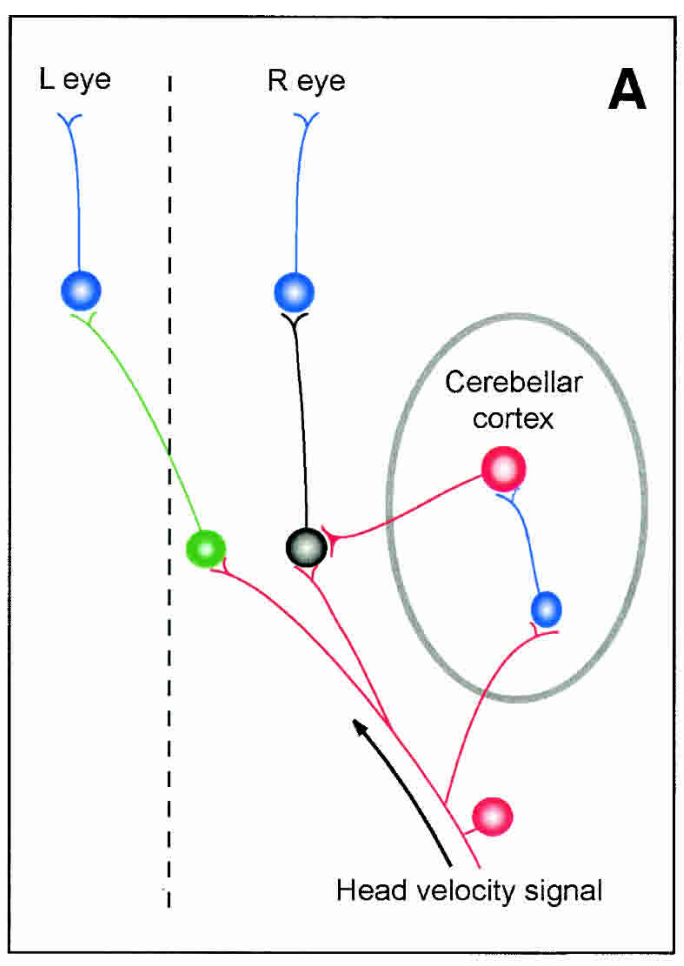

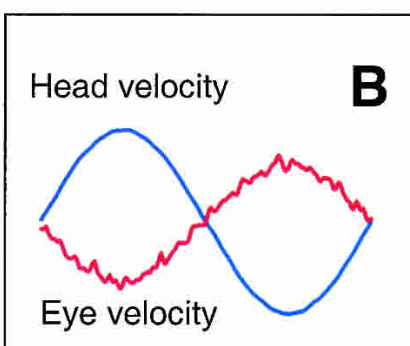

Firing rate

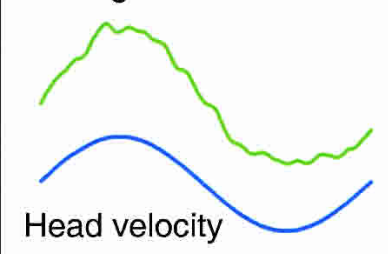

Head velocity
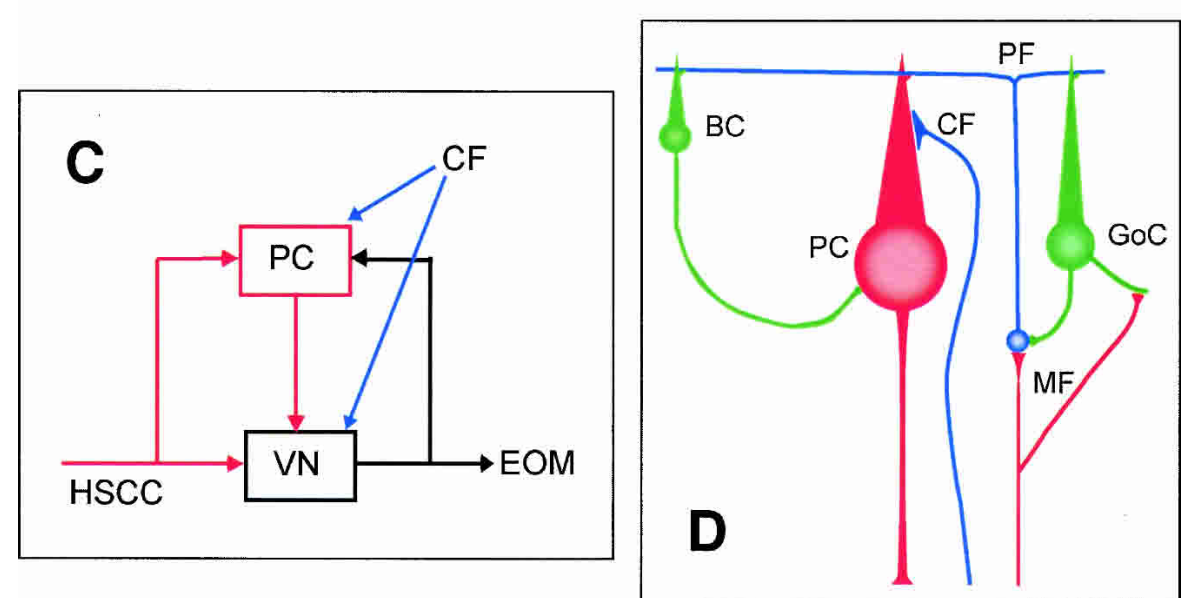

Figure 1 (A) Circuitry of the horizontal rotatory VOR. Primary afferents in the vestibular nerve (red) provide a head velocity signal to excitatory (green) and inhibitory (black) secondary vestibular neurons in the vestibular nuclei, which in turn project to motoneurons (blue) innervating the lateral rectus muscles. Because the axon of the excitatory interneuron crosses the midline (broken line) to the contralateral side, the excitatory and inhibitory pathways are synergistic. Additional pathways (not shown) innervate the medial rectus muscles. An inhibitory side loop modulates the VOR in situations where its gain is too high or too low for perfect stabilization. The side loop includes granule cells (blue) and Purkinje cells (red) of the vestibulocerebellum. The Purkinje cell output signal inhibits the inhibitory VOR interneuron. (B) Response of the VOR to rotation. Head (blue) and eye (red) movements are opposite in direction and approximately equal in velocity. Data from alert cat (gain $=0.8$ ). The firing rate of a secondary neuron (green) closely follows head velocity. (C) Signal flow in the cerebellar cortex and the VOR. Primary afferent fibers from the horizontal semicircular canal (HSCC) send head velocity signals to both the Purkinje cell and to the VOR interneuron in the vestibular nuclei (VN). The Purkinje cell in turn inhibits the VOR interneuron. Climbing fibers (CF) excite Purkinje cells and send collaterals to the vestibular nuclei as well. An appropriate eye velocity signal is sent to the extraocular muscles (EOM). A feedback loop provides a copy of the eye velocity signal (via mossy fibers) to the Purkinje cells. (D) Actual neural circuit of the cerebellar cortex. Input signals arrive in the cortex via mossy fibers (MF, red) and climbing fibers (CF, blue). Granule cell axons (blue, also called parallel fibers) excite dendritic spines of Purkinje cells (PC), Golgi cells (GoC), and inhibitory interneurons (BC or basket cell). Thus, excitatory inputs from climbing fibers and parallel fibers converge on the Purkinje cell dendrites. Redrawn from Eccles et al. 1967, with permission from Springer (0) 1967.

called VOR adaptation or plasticity. However, the terms "adaptation" and "plasticity" can also refer to short-term neural phenomena, whereas VOR motor learning is a long-lasting change.

VOR motor learning functions to improve performance, as quantified by two measures, the gain of the VOR (the ratio of eye to head speed) and its phase (the temporal relationship between eye and head movement). Figure 1B shows an example of the response to a sinusoidal rotation at $1 \mathrm{~Hz}$. Eye movements are opposite in direction and approximately equal in speed to the head movement. A gain of 1.0 and phase of $180^{\circ}$ would describe a perfect VOR for a purely horizontal head movement under normal conditions. In the laboratory, the optimal values of gain and phase depend on the stimulus conditions. Learning can be detected by measuring the gain of the VOR in darkness after the subject has been exposed to particular combinations of visual stimuli and head movement.

\section{The VOR Neural Network}

Most studies of VOR motor learning have focused on the eye movements evoked by the activation of the horizontal semicircular canals, that is, the horizontal rotatory VOR, although motor learning also takes place in the translational (Seidman et al. 1999) and vertical rotatory (Schultheis and Robinson 1981) VOR systems. Circuitry for the horizontal rotatory VOR is shown in Figure 1A. Primary afferent fibers from the horizontal semicircular canal (shown in blue) provide a head velocity (i.e., speed in a particular direction) signal to secondary neurons in the vestibular nuclei. The majority of secondary neurons increase their discharge rates as the head is rotated toward the side that they are on (Fig. 1B). Secondary neurons, also known as interneurons, project to the motoneurons, located in the abducens nuclei, which innervate the lateral rectus muscles. The direct projections are excitatory (green) to the contralateral and inhibitory (black) to the ipsilateral abducens. Therefore, the shortest VOR pathway is a 3-neuron arc within the brainstem. The medial rectus muscle is innervated by other projections (not shown). Additional components include the cerebellum, the nucleus prepositus hypoglossi, and the reticular formation. Most important for motor learning is the pathway through the flocculus and ventral paraflocculus of the cerebellum (lateral vestibulocerebellum), which terminates on the inhibitory VOR interneurons.

\section{Learning \& Memory www.learnmem.org}


The convergence of the three signals-head speed, eye speed, and visual image motion-which are important for VOR motor learning, occurs both in the cerebellar cortex and in the vestibular nuclei. Figure 1D illustrates the basic cerebellar cortical circuit, consisting of mossy (MF) and climbing fiber (CF) inputs, excitatory (granule cell, GrC), and inhibitory (basket cell, BC) interneurons, and Purkinje cells (PC; for more details, see Eccles et al. 1967). Purkinje cells are inhibitory and project to the vestibular nuclei (in the case of the lateral vestibulocerebellum). The cerebellar cortical circuit can be integrated into the VOR circuitry in a feedback arrangement, such as that shown in Figure 1C. Information about head movement arrives at the lateral vestibulocerebellum from the vestibular primary afferents and the vestibular nuclei (Langer et al. 1985; Nagao et al. 2002), by way of the mossy fiber/parallel fiber pathway (Miles et al. 1980b). At the same time, the cerebellar Purkinje cell receives climbing fiber input from the inferior olivary nucleus that encodes visual information (Maekawa and Simpson 1973). Information about ongoing smooth pursuit eye movements arrives at the lateral vestibulocerebellum via mossy fibers (Miles et al. 1980b) from the dorsolateral pontine nuclei (Mustari 1988), as well as from the vestibular nuclei. Eye-movement signals, including smooth pursuit and VOR-generated signals, are represented by the feedback pathway (black) in Figure 1C. Some mossy fibers also carry visual information (Noda 1986). Purkinje cells of the lateral vestibulocerebellum project directly back to the vestibular nuclei, this simple arrangement reflecting the early phylogenetic origin of the VOR circuitry.

\section{Theories and Early Experiments}

Even before motor learning had been demonstrated in the VOR, Masao Ito proposed that the cerebellar side loop might be the site for changes underlying the modification of reflexes (Ito 1972). The GABAergic projection from Purkinje cells to VOR interneurons (Fig. 1A) reduces the signal that is transmitted to motoneurons. Ito's hypothesis, based upon the ideas of Marr (1969) and Albus (1971), was that climbing fibers from the inferior olive provide a visual teacher signal that adjusts the sensitivity of the Purkinje cell to particular inputs from parallel fibers. Parallel fibers were assumed to provide a head-velocity signal. Due to plasticity at the parallel fiber/ Purkinje cell synapse, Purkinje cells would then become more sensitive to head velocity, decreasing the gain of the VOR, or less sensitive, increasing the gain. However, in thinking about Ito's hypothesis, it is important to keep in mind that the cerebellum is not the only site of convergence of visual and vestibular signals; such convergence also takes place in the brainstem.

Experimentally, learning can be imposed on the VOR by manipulating the visual image to induce apparent errors in the stabilization of gaze. Initially, reversing prisms were used as part of a portable headset (Gonshor and Melvill Jones 1973). Reversal of vision induces tremendous image motion on the retina, providing a strong (and quite disorienting) stimulus for motor learning, and inducing it rapidly and effectively. A less- disorienting visual stimulus is provided by miniaturizing and magnifying spectacles, or telescopes (Gauthier and Robinson 1975). In both cases, using the subject's own movements to provide the vestibular signal has the advantage of providing both stimuli with the correct frequency composition and the opportunity to use operant strategies. Figure 2 illustrates the exponential time course of motor learning. In monkeys wearing telescopic spectacles, the time constant of the exponential change in gain is different for increases (Fig. 2 top, left) and decreases in gain (Fig. 2 top, right), starting from the normal gain. During the return to normal without the telescopes, the difference disappears (Fig. 2, bottom two panels; Miles and Eighmy 1980).

If the subject is rotated passively, miniaturizing spectacles can decrease the gain of the VOR over a period of as little as 15 min. For lateral-eyed animals, lenses can be replaced with a highcontrast pattern that covers the entire visual field and can be rotated independently of the animal.

\section{Rules for the VOR and Motor Learning}

In evaluating potential mechanisms for VOR motor learning, it is helpful to remember a few principles that are generally agreed upon. These may be briefly summarized as follows: (1) an intact, functioning cerebellar cortex is required; (2) vision, in combination with another signal, is required; (3) the second signal can be either vestibular or oculomotor; and (4) both the lability and reversibility of VOR motor learning depend on the exact conditions and on the time course of learning and memory. VOR motor learning requires visual sensory input paired with either head or eye movement (Collewijn and Grootendorst 1979; Lisberger et al. 1984; Shelhamer et al. 1994). Specifically, the gain of the VOR will be increased if either (1) the head movement and retinal slip are in opposite directions, or (2) the eye movement and retinal

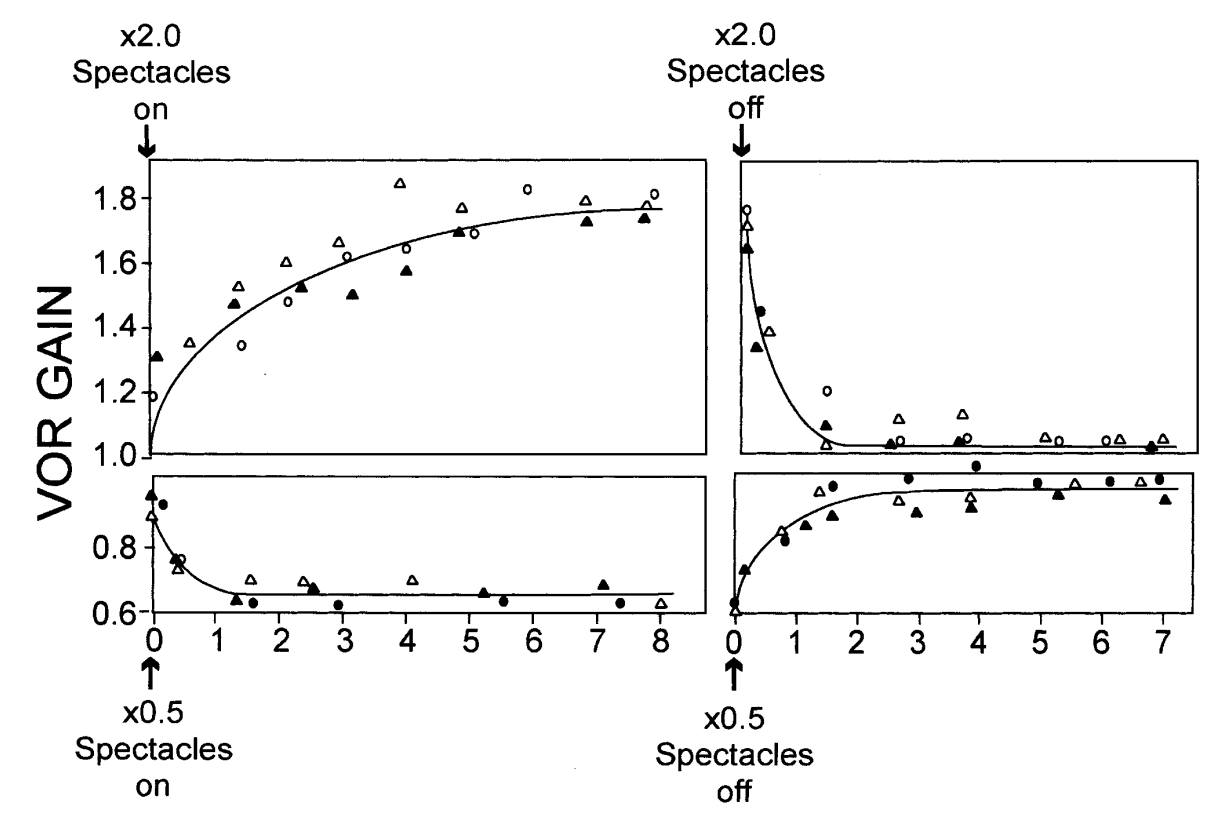

TIME (Days)

Figure 2 The time course of motor learning. Data are shown from monkeys wearing magnifying $(\times 2.0$, top two panels) or miniaturizing $(\times 0.5$, bottom) telescopic spectacles. Head velocity was provided by the monkey's own movements. Different symbols are from different animals. The change in gain was fit by an exponential decay. (Right) The spectacles were removed and the VOR gain was allowed to return to normal. The return to normal had a different time constant for high as compared with low gain. Redrawn from Miles and Eighmy (1980) with permission from The American Physiological Society @ 1980. 
slip are in the same direction. In the reverse of each of these situations, the gain of the VOR will be decreased. Depending on the site of learning, this rule can be translated into specific pairings of pre- and postsynaptic activity either in the cerebellum or in the vestibular nuclei (du Lac et al. 1995). The visual stimulus need not be full-field retinal slip; smooth pursuit alone, against a dark background, can change the gain of the VOR (Shelhamer et al. 1994), suggesting that visual signals from the small pursuit target are adequate for motor learning. In other words, parafoveal retinal slip paired with eye movement in the same direction can increase VOR gain. A strobe signal can even substitute for the pursuit target (Scudder and Fuchs 1992a), suggesting that visual motion per se is not necessary for learning.

Presentation of visual and vestibular stimuli can be broken into trials, in which the temporal relationships between rotation and vision can be varied (Raymond and Lisberger 1996), which is consistent with the associative model. However, there are important differences between the VOR and other forms of associative learning (for review, see Quinn 1998). For example, extinction does not occur reliably when either stimulus is presented alone (Miles and Eighmy 1980). It is also important to keep in mind that although motor learning is induced in the laboratory using optical methods, it is generally assumed that the system is plastic in order to deal with changes due to injury, disease, or aging in various components of the VOR, and learning can also be induced by peripheral vestibular damage (Maioli and Precht 1985; Broussard et al. 1999).

\section{Evidence for Sites of Learning and Memory}

Several laboratories have looked for evidence of changes in the cerebellar cortex that would support Ito's hypothesis. Early lesion studies showed unequivocally that the lateral vestibulocerebellum is required for motor learning (Ito et al. 1974; Robinson 1976; Lisberger et al. 1984). However, Miles and coworkers carried out a thorough investigation of ventral parafloccular Purkinje cell responses to rotation, only to conclude that although they change when motor learning occurs, they change in the wrong direction to support learning (Miles et al. 1980a). In the long term, Purkinje cells decrease their sensitivity to rotation when VOR gain decreases. This should have the effect of increasing the sensitivity of the interneurons, which would be expected to lead to an increase in VOR gain. This led to the proposal that the vestibular neurons themselves (by default) must be the site in which motor memory is stored by some other mechanism (Miles and Lisberger 1981). The Miles hypothesis introduced the idea that the cerebellar cortex, or the inferior olive, can provide a teacher signal to bring about modifications that are stored at a brainstem site. For example, the sensitivity of vestibular neurons to inputs from the vestibular commissure, or to polysynaptic as well as monosynaptic inputs from the ipsilateral vestibular nerve, could be altered by a Hebbian mechanism (du Lac et al. 1995). In support of this hypothesis, direct transmission by the 3-neuron arc was shown to be modified slightly during motor learning (Broussard et al. 1992; Khater et al. 1993).

Lisberger and coworkers showed that the VOR interneurons that receive Purkinje cell inputs modify their discharge patterns dramatically during motor learning; in fact, they change the polarity of their responses to rotation (Lisberger 1988; Lisberger et al. 1994b). In other words, when the VOR learns a lower gain, these neurons begin to increase their discharge rates during rotation away from the side they are on. Notably, this difference, like the difference in the VOR's response, was evident at short latencies after the start of a sudden rotation. In contrast, the differences in Purkinje cell responses were evident only at longer latencies (Lisberger et al. 1994a). The modifiable brainstem neu- rons are believed to be inhibitory VOR interneurons that project to the ipsilateral abducens nucleus (Broussard and Lisberger 1992; Scudder and Fuchs 1992b). The excitatory, contralaterally projecting secondary neurons probably remain unmodified during motor learning (Keller and Precht 1979; Lisberger and Miles 1981).

Concurrently, other investigators have continued to gather evidence in support of Ito's hypothesis. Purkinje cell responses during the VOR, when both head and eye velocity signals contribute to their responses, were shown to be modified in a way that would support motor learning (Watanabe 1984; Nagao 1989). The modification of Purkinje cell responses in the correct direction to support learning may have arisen from brainstem changes by way of the feedback loop shown in Figure 1C (black lines), as their latencies suggested (Lisberger et al. 1994a). However, the fact that models of the VOR become unstable when only a single site in the brainstem is permitted to change (Lisberger 1994) argued in favor of cerebellar cortical plasticity in addition to the brainstem site. Changes in dynamics of cerebellar inputs were also required in order to generate realistic Purkinje cell responses in the model (Lisberger and Sejnowski 1992; Lisberger 1994).

Studies on VOR motor learning have been compared directly with studies on the timing of the eyeblink reflex evoked by a conditioned stimulus. Although the experimental paradigms are very different, the results are consistent across the two systems. The conclusions from both can be summarized very simply: Changes in the cerebellar cortical circuitry are responsible, in the long term, for appropriate timing of learned responses (Perrett et al. 1993; Raymond et al. 1996), whereas changes in the deep cerebellar nuclei and vestibular nuclei are necessary for expression of conditioned responses (du Lac et al. 1995; Raymond et al. 1996; Mauk 1997). We will argue in a later section for a modified version of this hypothesis.

\section{Possible Cellular Mechanisms in the Cerebellum and Brain Stem}

Cellular mechanisms that have been proposed for VOR motor learning include Hebbian and non-Hebbian synaptic plasticity, as well as postsynaptic changes in excitability (in Hebbian synaptic plasticity, a synapse is strengthened after the pre- and postsynaptic neuronal discharges are correlated for a period of time). We will review briefly first cerebellar, then brainstem mechanisms.

\section{Cerebellar Mechanisms}

The best-documented type of synaptic plasticity in the VOR circuitry is cerebellar long-term depression (LTD). Cerebellar LTD requires a coincidence of parallel fiber and climbing fiber input. In LTD, the excitatory synapse from parallel fibers to Purkinje cells is depressed following coactivation of parallel and climbing fibers, or following climbing fiber stimulation paired with glutamate application (Ito et al. 1982; Linden and Connor 1995). Climbing fiber activation causes an increase in calcium influx into the Purkinje cell dendrite through voltage-gated channels, whereas parallel fiber inputs activate metabotropic and AMPA types of glutamate receptors. Protein kinase $\mathrm{C}$ is activated and nitric oxide is released in subsequent steps (Daniel et al. 1998; for review, see Hansel et al. 2001).

LTD can be induced in the flocculus by concurrently activating climbing fiber inputs and mossy fiber vestibular inputs (Ito et al. 1982). This mechanism would presumably reduce Purkinje cell sensitivity to rotation in situations requiring an increase in VOR gain, because retinal slip signals in the direction of rotation would arrive by way of the climbing fibers, concurrently 
with vestibular signals in the same direction from primary vestibular afferents. Thus, cerebellar LTD provides a reasonable mechanism for associative motor learning. Strong support for this idea has been obtained recently. Inactivation of cerebellar LTD by inhibition of protein kinase $C$ in the Purkinje cells of a transgenic mouse prevented optically induced motor learning in the VOR (de Zeeuw et al. 1998).

VOR gain is capable of decreases as well as increases; one convenient way in which this could be mediated by cerebellar cortical learning would be to impose LTD and long-term potentiation (LTP) at the same synapse, reversing each other. However, LTP at the parallel fiber-Purkinje cell synapse is thought to operate primarily by a presynaptic mechanism, implying that it cannot reverse LTD (Salin et al. 1996). This would not allow repeated increases and decreases in VOR gain, which are known to be possible (for example, see Broussard and Hong 2003). More recently, a postsynaptic form of cerebellar LTP has also been described (Lev-Ram et al. 2002).

Other evidence indicates that increases and decreases in gain are fundamentally different. After several days of wearing telescopes, gain increases, but not decreases, would return slowly toward normal in the absence of head movement (Miles and Eighmy 1980). Time courses tend to be different for increases and decreases (Fig. 2). More recent studies have shown that gain increases are also more easily reversible than gain decreases immediately after learning in mice (Boyden and Raymond 2003). Taken together, the results suggest that it would be consistent with a scenario in which distributed pre- and postsynaptic LTP is responsible for gain decreases, and postsynaptic LTD for gain increases (Boyden and Raymond 2003). Because LTP is distributed across two sites, it would not be completely reversed by postsynaptic LTD, but LTD could be completely reversed by LTP. In the goldfish, there is more direct evidence that gain increases and decreases utilize different cellular mechanisms. The intercellular messenger nitric oxide (which is thought to be important for LTD) is required for gain increases, but not decreases (Li et al. 1995), and metabotropic and $N$-methyl-D-aspartate (NMDA)type glutamate receptors in the cerebellar cortex also appear to participate in gain increases, but not decreases (for review, see McElligott and Spencer 2000).

Although in most models of motor learning, only the parallel fiber-Purkinje cell synapse is plastic, in fact, many of the other synapses in the cerebellar network, as well as the synapse between the Purkinje cell and its postsynaptic target (Kano 1996; Aizenman et al. 1998; Ouardouz and Sastry 2000), are capable of long-term changes (for review, see Hansel et al. 2001). Many such sites have been excluded from models because they yield no obvious behavioral advantage, but the sheer number of types of synaptic plasticity discovered so far indicates the need for more flexible thinking on this issue.

\section{Brainstem Mechanisms}

In the brainstem, Grassi and coworkers have demonstrated LTP of the field potentials presumably arising from the excitatory synapse between primary afferents and secondary vestibular neurons (Fig. 1; see Grassi and Pettorossi 2001 for a recent summary). The potentiation depends on NMDA receptor activation (Capocchi 1992) and can be reversed by low-frequency stimulation (Grassi et al. 1996).

The interneurons in the VOR that receive cerebellar inhibition are known as flocculus target neurons (FTNs; Fig. 1A, black neuron). FTNs receive direct inputs from the vestibular nerve, so that LTD or LTP of the primary afferent-secondary neuron synapse could provide an explanation for the changes in FTN re- sponses to rotation. However, in experiments in which the VOR pathway was activated by electrical stimulation before and after motor learning, the response of the 3-neuron arc was modified very little (Broussard et al. 1992; Broussard and Hong 2003). This could reflect the relatively small number of FTNs, but it is still important to consider additional sites of synaptic plasticity in the vestibular nuclei. After the horizontal semicircular canal is plugged unilaterally, recovery involves changes in commissural inputs to vestibular neurons (shown in Fig.1A; Farrow and Broussard 2003), and a similar mechanism could operate in motor learning.

In addition to synaptic plasticity, changes in the excitability of secondary vestibular neurons provide additional possible mechanisms for altering the gain of the VOR pathway (du Lac 1996). A sustained increase in excitability of deep cerebellar nuclear neurons has been reported following tetanization of afferent pathways (Aizenman and Linden 2000), and an analogous mechanism could operate in the vestibular nuclei. Both SK and BK-type calcium-dependent potassium channels have been implicated recently as sites for long-term changes in excitability (and potentially for memory) in the vestibular nuclei (Nelson et al. 2003).

\section{Consolidation of VOR Motor Memory}

Like other kinds of memory, motor memory undergoes a process of consolidation. For the purpose of this review, we will define consolidated memory as one that is relatively resistant to disruption. This definition was originally based on studies in which a disruptive event, such as trauma or electroconvulsive therapy, disrupted previously existing memories, particularly recent ones; recent memories were taken to be less consolidated (for example, see Squire et al. 1975). Consolidation usually differentiates shortterm from long-term memory and may involve a change in the locus of memory storage (Shadmehr and Holcomb 1997). There have been only a few investigations of the consolidation process in the VOR; most studies have described long-term motor learning, over a period of days to weeks, in which memory is almost certainly consolidated. Unpaired visual or vestibular stimulation can extinguish long-term memory in the VOR in some cases (Miles and Eighmy 1980). Therefore, all results that pertain to consolidated (or long-term) memory must be compared with the corresponding short-term outcome.

When investigators have inactivated the lateral vestibulocerebellum in an attempt to abolish previously acquired motor memory, results have varied in a way that suggests a consolidation process. Repetitive climbing fiber stimulation, which causes floccular shutdown, had no effect on changes in VOR gain that were learned over several days (Luebke and Robinson 1994), suggesting that the cerebellar cortex does not participate in the longterm storage of motor memory. Unilateral inactivation of the lateral vestibulocerebellum in monkeys also did not abolish longterm memory in the vertical VOR (Partsalis et al. 1995). However, in the goldfish, McElligott and coworkers (McElligott et al. 1998) were able to completely abolish changes in gain that had been learned within the previous few hours, by infusing lidocaine into the flocculus. More recently, similar results were obtained in the monkey (Nagao and Kitazawa 2003). Both results are consistent with the interpretation that the cerebellar cortex is the exclusive site of storage.

A possible explanation of these different outcomes is the transfer hypothesis (Galiana 1986; Peterson et al. 1991; Raymond et al. 1996). In the transfer hypothesis, short-term changes may take place in the cerebellar cortex, after which the cortex generates an appropriate error signal to drive the formation of longterm memory in the brainstem. A similar idea, the trigger-and- 
storage hypothesis, has been proposed for the eyeblink conditioned reflex and for fear conditioning (Mauk 1997; Medina et al. 2002). Experimental evidence has provided some support for memory transfer in the eyeblink system (Ohyama and Mauk 2001; Attwell et al. 2002). The trigger-and-storage hypothesis was used as the basis for the model in Figure 3B (see below).

The distinction between short-term and long-term motor memory in the VOR was made clear by de Zeeuw and coworkers, who created transgenic mice that were deficient in LTD at the Purkinje cell-parallel fiber synapse. The transgenic mice could not adapt their VOR gain during short-term motor learning (de Zeeuw et al. 1998), although Purkinje cells responded to visual and vestibular stimuli during situations that would be expected to evoke motor learning (Goossens et al. 2001). This suggested that the most effective mechanism for short-term motor learning requires cerebellar cortical LTD. However, the results also suggested that LTD is not required for all motor learning, because gain changes were expressed after learning, lasting $8 \mathrm{~d}$ (van $\mathrm{Al}-$ phen and de Zeeuw 2002). One possible interpretation is that two mechanisms exist for short-term learning, one requiring LTD and one not requiring it, and that either can contribute (through consolidation) to long-term memory.

To test the hypothesis that short-term motor memory is stored primarily in the cerebellar cortex, we partially inactivated the flocculus and ventral paraflocculus bilaterally in cats, immediately after $1 \mathrm{~h}$ of motor learning (short-term paradigm; Kassardjian et al. 2003). The pharmacological agent used, 6-cyano-7nitroquinoxaline-2,3-dione (CNQX), is an AMPA/kainate receptor antagonist that blocks all excitatory input to Purkinje cells. The CNQX injections were compared with injections of the buffered saline vehicle on alternate days. In a second experiment, we made the same bilateral CNQX injections in cats after 3 $\mathrm{d}$ of motor learning (long-term paradigm) and compared the gains with the preinjection values.

The results are shown in Figure 3A. In the short-term paradigm, the VOR gain measured at $2 \mathrm{~Hz}$ decreased significantly during learning. Saline injections did not produce any significant changes in the post-motor learning VOR gain. After bilateral injections of CNQX, eye velocity returned to the pre-adaptation value and was significantly different from the post-adaptation value $(P<0.001, n=4$, unpaired $t$-test $)$. A different outcome was observed in the long-term paradigm in the same cat. In this experiment, the VOR was adapted to low gain over a 3-day period, and then CNQX was injected. The adapted gain increased slightly, but the result was not highly significant $(P>0.03, n=4$, unpaired $t$-test). We concluded that in the long term, the cerebellar cortex contributed less to the storage of motor memory than it did in the short term. This idea is illustrated by the diagrams in Figure $3 \mathrm{~B}$, which were based loosely on the trigger-and-storage hypothesis of Mauk and coworkers (Medina et al. 2002). In the left diagram, the shortterm trigger involves changes in the cerebellar cortex. During consolidation (right), changes occur in the vestibular nuclei.

\section{Can Learning Be Applied to Different Stimuli?}

In some situations, it would be useful to apply motor learning to stimuli that did not occur during training; however, universal application of the acquired motor fiber signals induce learning in the vestibular nuclei (VN).

\section{Learning \& Memory}


memory could also be a disadvantage. For example, weakness of one extraocular muscle would require the VOR to adjust its gain centrally, but only for movements in which that muscle participates. Evidence suggests that motor learning in the VOR may be selective for both the direction and the frequency of rotation at which training occurred (Lisberger et al. 1983; Raymond and Lisberger 1996; Hirata et al. 2002). We will use the VOR's response to rotation at different frequencies to illustrate this issue. Accurate responses to rapidly changing stimuli by the VOR are especially important for good vision during locomotion, because visual mechanisms for gaze stabilization are extremely limited in their response at high frequencies; the importance of the VOR to the organism probably increases with stimulus frequency in the $0.1-10 \mathrm{~Hz}$ range. However, the error signal for VOR motor learning is visual, and therefore subject to the relatively slow response of the visual system. At the level of the Purkinje cell, increases in the stimulus frequency cause phase lags in the putative error signals in the cerebellar cortex (Raymond and Lisberger 1998). Lags in visual (but not vestibular) signals would prevent the coincidence of pre- and postsynaptic signals that is necessary in many models of learning. In addition, at high frequencies, gaze is poorly stabilized during rotation in the light, leading to different patterns of retinal slip compared with those at low frequencies.

Given the frequency limitations of some error signals, it is perhaps not surprising that motor learning does not generalize to the VOR at frequencies for which it was not trained. For example, restriction of rotation to only high frequencies during learning results in more learning at high than at low frequencies (Lisberger et al. 1983; Raymond and Lisberger 1996). This outcome has been used to argue for the existence of frequency channels, or processing streams in the VOR (Lisberger et al. 1983). A correlate of frequency streams might be the existence of different mechanisms for motor learning at different frequencies. Documentation of simple and complex spike signals in Purkinje cells during rotation at different frequencies revealed that at 5 and $10 \mathrm{~Hz}$, a comparison of Purkinje cell discharge patterns and climbing fiber inputs (which happens at the Purkinje cell) could be used to guide learning, whereas at lower frequencies, Purkinje cell discharges and vestibular signals can be compared with guide learning (Raymond and Lisberger 1998); this would seem more likely to occur in the brainstem.

There is some evidence that high- and low-frequency, VOR circuitry may also use different molecular machinery. Blockade of $\mathrm{N}$-methyl-D-aspartate (NMDA) receptors has a selective effect on the low-frequency VOR, which is not duplicated by blockade of other types of ionotropic glutamate receptors (Priesol et al. 2000). NMDA receptors have a known role in synaptic plasticity in many systems and also are known to contribute to the commissural connections between the vestibular nuclei on the two sides of the brainstem (Knöpfel 1987; Doi et al. 1990). Selective transmission of low-frequency VOR signals by the brainstem pathways that contain active NMDA receptors might mean that NMDA-dependent mechanisms in the brainstem can operate preferentially on low-frequency VOR signals. More investigation will be required to establish whether different mechanisms and/ or different input pathways are responsible for the segregation of motor learning at different frequencies.

\section{Comparison of Model Species and Future Directions}

The vestibular nuclei and flocculus are two of the phylogenetically most-ancient parts of the vertebrate brain. Even in lampreys, vestibular nuclei are linked to the primitive cerebellum (Sarnat and Netsky 1974). Motor learning in the VOR has been shown to exist and operate over a similar time course in humans (Gonshor and Melvill Jones 1976), rabbits (Ito et al. 1974), ma- caques (Miles and Eighmy 1980), squirrel monkeys (Clendaniel et al. 2002), cats (Robinson 1976), mice (de Zeeuw et al. 1998), and goldfish (Pastor et al. 1992). In general, the anatomy of the VOR pathway is strikingly similar across all of the species to be discussed, with relatively minor differences such as the size of the oculomotor nuclei and the exact locations of subpopulations of neurons.

The advantage of using the mammalian brain to study learning may seem obvious; whatever we learn can be applied to ourselves with more confidence, and behaviors that exist in humans can often be studied (like the VOR). However, the optimal species model depends on the experimental paradigm. In most cases in which eye movements are to be measured, monkeys, cats, or rabbits are preferred because the large size of the eye permits the attachment of scleral search coils for accurate, reliable eye position records. Although there are visual differences (e.g., placement and size of the eyes, photoreceptor type, structure of the retina), differences in the circuitry of the VOR itself among rodents, cats, and monkeys are relatively minor.

Rhesus monkeys (Macaca mulatta) and other macaques are excellent behavioral and neurophysiological models due to their accurate VOR, rapid, highly consistent motor learning, and suitability for chronic electrophysiology. Because they possess a fovea, primates can be trained to perform accurate saccadic and smooth pursuit eye movements, which (in this context) serve as useful tools for understanding signal processing by the VOR circuitry. Motor learning in the rhesus exhibits some idiosyncratic behavior compared with other species; for example, in the rabbit (Collewijn and Grootendorst 1979) and human (Shelhamer et al. 1994), VOR motor learning can be induced without any vestibular sensory input, but in the rhesus, rotation is required (Lisberger et al. 1984). However, such interspecific differences in the details of learning can provide valuable information about the constraints on motor learning mechanisms.

Rhesus monkeys are expensive, difficult to obtain, and subject to ethics and health issues. They cannot be used effectively for invasive experiments that require large numbers of animals, ex vivo slice work (or any kind of cellular neurophysiology), or for development of transgenics. Squirrel monkeys (Saimiri sciurius), another popular model, were used in early vestibular nerve studies (Fernandez and Goldberg 1971; Goldberg and Fernandez 1971) and for cellular electrophysiology (McCrea et al. 1987), and are superior to the rhesus when surgical access to the labyrinth is required. However, squirrel monkeys are generally less robust and more difficult to train than the rhesus.

Domestic cats (Felis catus) have been a popular model for anatomical and physiological studies, including intracellular recordings, and the VOR pathway is well known in this species. The advantages of using cats are the ease and relatively low cost of obtaining animals, their durability and ease of handling, the large size of the eye, and the ease of surgical access to the inner ear. The cat has an area centralis, frontal eyes, relatively good vision, and a VOR gain similar to the human. However, VOR motor learning is less effective in cats compared with monkeys (Luebke and Robinson 1994), and cats are difficult to train to produce saccadic and smooth pursuit eye movements with the head fixed. There are also differences in the mean discharge rates of secondary vestibular neurons in the cat and primate (Keller and Precht 1979), which may turn out to be important for signal processing in the VOR.

Rabbits (Oryctolagus sp.) were used in many early studies of VOR motor learning (Maekawa and Simpson 1973; Ito et al. 1974; Collewijn and Grootendorst 1979) and vestibular circuitry (Highstein et al. 1971; Highstein 1973). The rabbit has poor visual acuity and the gain of the VOR is naturally low. Some strains of rabbits have markedly abnormal eye movements (Collewijn 
and Grootendorst 1978). However, interspecific differences in the details of learning, such as the interaction (or lack of it) with saccadic and smooth pursuit systems, can still provide valuable information.

Rodents, including chinchillas (Chinchilla laniger), guinea pigs (Cavia porcellus), jirds or Mongolian gerbils (Meriones unguiculatus), rats (Rattus norvegicus), and mice (Mus musculus) have been used in many investigations of the vestibular system. One major advantage of rodent models is that ex vivo preparations, such as the brainstem slice (Lewis et al. 1987), can be developed to investigate cellular neurophysiology. In the case of the mouse, transgenic strains are also available, including many neurotransmitter receptor, metabolic and ion channel mutants, and one strain in which floccular Purkinje cell terminals are fluorescent (Sekirnjak et al. 2003). Transgenics have already provided invaluable information regarding cellular mechanisms for motor learning (de Zeeuw et al. 1998; Shutoh et al. 2002).

On the other hand, rodents present some disadvantages for behavioral experiments. Some do not accept restraint easily and quickly become stressed. The eyes are lateral, vision poor, saccades and smooth pursuit absent; the gain of the VOR is naturally low in most rodents, and scleral search coils are difficult to implant in the smaller species. Coils can be glued to the cornea, which presents its own problems (Stahl et al. 2000; van Alphen et al. 2001). Recently, however, image tracking systems have been used successfully in mice, revealing a surprisingly high VOR gain (Stahl et al. 2000), and VOR motor learning has also been documented in this species (de Zeeuw et al. 1998; Boyden and Raymond 2002).

Most nonmammalian vertebrates are active during daylight hours and rely on vision. In contrast, with the exception of humans, their domestic animals, and other primates, most mammals are nocturnal (Hairston 1994, p.68). Consequently, there are more examples among nonmammals of species with good visual acuity, high VOR gain, excellent motor learning and an expanded oculomotor repertoire. The only nonmammalian model that has been used in a significant number of studies of VOR motor learning is the goldfish. Because fish are not generally in direct contact with a firm substrate and cannot move their heads, gaze stabilization in fish must rely exclusively on visual and vestibular signals reaching the oculomotor system. Goldfish have high VOR gains and excellent VOR motor learning, and have been used successfully to demonstrate storage of short-term VOR motor learning in the cerebellar cortex (McElligott et al. 1998) and monocular motor learning (McElligott and Wilson 2001). The fish cerebellar circuitry exhibits some differences from mammals (Larsell 1970). Therefore, it is possible that major differences will appear as the goldfish VOR is further studied and compared with mammals.

To summarize, it appears that no one model is best for investigating all aspects of VOR motor learning. Instead, multiple models should be used, depending on the exact issues to be addressed. The use of multiple models is further encouraged by the fact that mechanisms are likely to be similar in this relatively conserved motor system.

\section{ACKNOWLEDGMENTS}

We thank M. Peterson and Y.-F. Tan for technical assistance, and A. Guy for critical comments on the manuscript. This work was supported by operating grants from CIHR and NSERC of Canada.

\section{REFERENCES}

Aizenman, C.D. and Linden, D.J. 2000. Rapid, synaptically driven increases in the intrinsic excitability of cerebellar deep nuclear neurons. Nat. Neurosci. 3: 109-111.

Aizenman, C.D., Manis, P.B., and Linden, D.J. 1998. Polarity of long-term synaptic gain change is related to postsynaptic spike firing at a cerebellar inhibitory synapse. Neuron 21: 827-835.

Albus, J.S. 1971. A theory of cerebellar function. Math. Biosci. 10: 25-61.

Attwell, P.J.E., Cooke, S.F., and Yeo, C.H. 2002. Cerebellar function in consolidation of a motor memory. Neuron 34: 1011-1020.

Boyden, E.S. and Raymond, J.L. 2003. Active reversal of motor memories reveals rules governing memory encoding. Neuron 39: 1031-1042.

Broussard, D.M. and Hong, J.A. 2003. The response of vestibuloocular reflex pathways to electrical stimulation after canal plugging. Exp. Brain Res. 149: 237-248.

Broussard, D.M. and Lisberger, S.G. 1992. Vestibular inputs to brainstem neurons that participate in motor learning in the primate vestibuloocular reflex. J. Neurophysiol. 68: 577-580.

Broussard, D.M., Bronte-Stewart, H.M., and Lisberger, S.G. 1992. Expression of motor learning in the response of the primate vestibuloocular reflex pathway to electrical stimulation. $J$ Neurophysiol. 67: 1493-1507.

Broussard, D.M., Bhatia, J.K., and Hong, J.A. 1999. The dynamics of the vestibulo-ocular reflex after peripheral vestibular damage. II. Comparison with dynamics after optically-induced learning. Exp. Brain Res. 125: 365-374.

Capocchi, G. 1992. NMDA receptor-mediated long term modulation of electrically evoked field potentials in the rat medial vestibular nuclei. Exp. Brain Res. 90: 546-550.

Clendaniel, R.A., Lasker, D.M., and Minor, L.B. 2002. Differential adaptation of the linear and nonlinear components of the horizontal vestibuloocular reflex in squirrel monkeys. J. Neurophysiol. 88: $3534-3540$.

Collewijn, H. and Grootendorst, A.F. 1978. Adaptation of the rabbit's vestibulo-ocular reflex to modified visual input: Importance of stimulus conditions. Arch. Ital. Biol. 116: 273-280.

. 1979. Adaptation of optokinetic and vestibulo-ocular reflexes to modified visual input in the rabbit. Prog. Brain Res. 50: 771-781.

Daniel, H., Levenes, C., and Crepel, F. 1998. Cellular mechanisms of cerebellar LTD. Trends in Neurosciences (TINS) 21: 401-407.

de Zeeuw, C.I., Hansel, C., Bian, F., Koekkoek, S.K.E., van Alphen, A.M., Linden, D.J., and Oberdick, J. 1998. Expression of a protein kinase C inhibitor in Purkinje cells blocks cerebellar LTD and adaptation of the vestibulo-ocular reflex. Neuron 20: 495-508.

Doi, K., Tsumoto, T., and Matsunaga, T. 1990. Actions of excitatory amino acid antagonists on synaptic inputs to the rat medial vestibular nucleus: An electrophysiological study in vitro. Exp. Brain Res. 82: 254-262.

du Lac, S. 1996. Candidate cellular mechanisms of vestibulo-ocular reflex plasticity. Ann. NY. Acad. Sci. 781: 489-498.

du Lac, S., Raymond, J.L., Sejnowski, T.J., and Lisberger, S.G. 1995. Learning and memory in the vestibulo-ocular reflex. Annu. Rev. Neurosci. 18: 409-442.

Eccles, J.C., Ito, M., and Szenthagothai, J. 1967. The cerebellum as a neuronal machine. Springer, Berlin, Germany.

Farrow, K. and Broussard, D.M. 2003. Commissural inputs to secondary vestibular neurons in alert cats after canal plugs. J. Neurophysiol. 89: 3351-3353.

Fernandez, C. and Goldberg, J.M. 1971. Physiology of peripheral neurons innervating semicircular canals of the squirrel monkey. II. Response to sinusoidal stimulation and dynamics of peripheral vestibular systems. J. Neurophysiol. 34: 661-675.

Galiana, H.L. 1986. A new approach to understanding adaptive visual-vestibular interactions in the central nervous system. $J$. Neurophysiol. 55: 349-374.

Gauthier, G.M. and Robinson, D.A. 1975. Adaptation of the human vestibuloocular reflex to magnifying lenses. Brain Res. 92: 331-335.

Goldberg, J.M. and Fernandez, C. 1971. Physiology of peripheral neurons innervating semicircular canals of the squirrel monkey. I. Resting discharge and response to constant angular accelerations. $J$. Neurophysiol. 34: 635-660.

Gonshor, A. and Melvill Jones, G. 1973. Changes of human vestibulo-ocular response induced by vision-reversal during head rotation. J. Physiol. 234: 102P.

. 1976. Extreme vestibulo-ocular adaptation induced by prolonged optical reversal of vision. J. Physiol. 256: 381-414.

Goossens, J., Daniel, H., Rancillac, A., van der Steen, J., Oberdick, J., Crepel, F., De Zeeuw, C.I., and Frens, M.A. 2001. Expression of protein kinase $\mathrm{C}$ inhibitor blocks cerebellar long-term depression without affecting Purkinje cell excitability in alert mice. J. Neurosci. 21: $5813-5823$.

Grassi, S. and Pettorossi, V.E. 2001. Synaptic plasticity in the medial vestibular nuclei: Role of glutamate receptors and retrograde messengers in rat brainstem slices. Prog. Neurobiol. 64: 527-553.

Grassi, S., Pettorossi, V.E., and Zampolini, M. 1996. Low-frequency stimulation cancels the high-frequency-induced long-lasting effects in the rat medial vestibular nuclei. J. Neurosci. 16: 3373-3380.

\section{Learning \& Memory}


Hairston, N.G.S. 1994. Vertebrate Zoology: An experimental field approach. Cambridge University Press, New York.

Hansel, C., Linden, D.J., and D'Angelo, E. 2001. Beyond parallel fiber LTD: The diversity of synaptic and non-synaptic plasticity in the cerebellum. Nat. Neurosci. 4: 467-475.

Highstein, S.M. 1973. Synaptic linkage in the vestibulo-ocular and cerebello-vestibular pathways to the VIth nucleus in the rabbit. Exp. Brain Res. 17: 301-314.

Highstein, S.M., Ito, M., and Tsuchiya, T. 1971. Synaptic linkage in the vestibulo-ocular reflex pathway of rabbit. Exp. Brain Res. 13: 306-326.

Hirata, Y., Lockard, J.M., and Highstein, S.M. 2002. Capacity of vertical VOR adaptation in squirrel monkey. J. Neurophysiol. 88: 3194-3207.

Horridge, G.A. 1962. Learning of leg position by the ventral nerve cord in headless insects. Proc. Royal Acad. Sci. Lond. B 157: 33-52.

Huterer, M. and Cullen, K.E. 2002. Vestibuloocular reflex dynamics during high-frequency and high-acceleration rotations of the head on body in rhesus monkey. J. Neurophysiol. 88: 13-28.

Ito, M. 1972. Neural design of the cerebellar motor control system. Brain Res. 40: $81-84$

Ito, M., Shiida, T., Yagi, N., and Yamamoto, M. 1974. The cerebellar modification of rabbit's horizontal vestibulo-ocular reflex induced by sustained head rotation combined with visual stimulation. Proc. Japan Acad. 50: 85-89.

Ito, M., Sakurai, M., and Tongroach, P. 1982. Climbing fibre induced depression of both mossy fibre responsiveness and glutamate sensitivity of cerebellar Purkinje cells. J. Physiol. 324: 113-134.

J.C. 1952. Living without a balancing mechanism. New Engl. J. Med. 246: 458-460.

Kano, M. 1996. Long-lasting potentiation of GABAergic inhibitory synaptic transmission in cerebellar Purkinje cells: Its properties and possible mechanisms. Behav. Brain Sci. 19: 354-361.

Kassardjian, C.D., Tan, Y.-F., and Broussard, D.M. 2003. Effects of partial reversible inactivation of the floccular complex on motor memory in the vestibulo-ocular reflex of the cat. Program No. 882.9. http://sfn.scholarone.com

Keller, E.L. and Precht, W. 1979. Adaptive modification of central vestibular neurons in response to visual stimulation through reversing prisms. J. Neurophysiol. 42: 896-911.

Khater, T.T., Quinn, K.J., Pena, J., Baker, J.F., and Peterson, B.W. 1993 The latency of the cat vestibulo-ocular reflex before and after shortand long-term adaptation. Exp. Brain Res. 94: 16-32.

Knöpfel, T. 1987. Evidence for N-methyl-D-aspartic acid receptor-mediated modulation of the commissural input to central vestibular neurons of the frog. Brain Res. 426: 212-224.

Langer, T., Fuchs, A.F., Scudder, C.A., and Chubb, M.C. 1985. Afferents to the flocculus of the cerebellum in the rhesus macaque as revealed by retrograde transport of horseradish peroxidase. J. Comp. Neurol. 235: $1-15$.

Larsell, O. 1970. The comparative anatomy and histology of the cerebellum from monotremes through apes. University of Minnesota Press, Minneapolis, MN.

Lev-Ram, V., Wong, S.T., Storm, D.R., and Tsien, R.Y. 2002. A new form of cerebeller long-term potentiation is postsynaptic and depends on nitric oxide but not cAMP. Proc. Natl. Acad. Sci. 99: 8389-8393.

Lewis, M.R., Gallagher, J.P. and Shinnick-Gallagher, P.S. 1987. An in vitro brain slice preparation to study the pharmacology of central vestibular neurons. J. Pharmacol. Methods 18: 267-273.

Li, J., Smith, S.S., and McElligott, J.G. 1995. Cerebellar nitric oxide is necessary for vestibulo-ocular reflex adaptation, a sensorimotor model of learning. J. Neurophysiol. 74: 489-494.

Linden, D.J. and Connor, J.A. 1995. Long-term synaptic depression. Annu. Rev. Neurosci. 18: 319-357.

Lisberger, S.G. 1988. The neural basis for learning of simple motor skills. Science 242: 728-735.

. 1994. Neural basis for motor learning in the vestibulo-ocular reflex of primates: III. Computational and behavioral analysis of the sites of learning. J. Neurophysiol. 72: 974-998.

Lisberger, S.G. and Miles, F.A. 1981. Role of primate medial vestibular nucleus in long-term adaptive plasticity of vestibuloocular reflex. $J$. Neurophysiol. 45: 869-890.

Lisberger, S.G. and Sejnowski, T.J. 1992. Motor learning in a recurrent network model based on the vestibuloocular reflex. Nature 360: $159-161$.

Lisberger, S.G., Miles, F.A., and Optican, L.M. 1983. Frequency-selective adaptation: Evidence for channels in the vestibulo-ocular reflex? $\mathrm{J}$. Neurosci. 3: 1234-1244.

Lisberger, S.G., Miles, F.A., and Zee, D.S. 1984. Signals used to compute errors in monkey vestibulo-ocular reflex: Possible role of flocculus. J. Neurophysiol. 52: 1140-1153.

Lisberger, S.G., Pavelko, T.A., Bronte-Stewart, H.M., and Stone, L.S. 1994a. Neural basis for motor learning in the vestibulo-ocular reflex of primates: II. Changes in the responses of horizontal gaze velocity Purkinje cells in the cerebellar flocculus and ventral paraflocculus. $J$. Neurophysiol. 72: 954-973.

Lisberger, S.G., Pavelko, T.A., and Broussard, D.M. 1994b. Neural basis for motor learning in the vestibulo-ocular reflex of primates: I. Changes in the responses of brain stem neurons. J. Neurophysiol. 72: $928-953$.

Luebke, A.E. and Robinson, D.A. 1994. Gain changes of the cat's vestibulo-ocular reflex after flocculus deactivation. Exp. Brain Res. 98: $379-390$.

Maekawa, K. and Simpson, J.I. 1973. Climbing fiber responses evoked in vestibulocerebellum of rabbit from visual system. J. Neurophysiol. 36: 649-666.

Maioli, C. and Precht, W. 1985. On the role of vestibulo-ocular reflex plasticity in recovery after unilateral peripheral vestibular lesions. Exp. Brain Res. 59: 267-272.

Marr, D. 1969. A theory of cerebellar cortex. J. Physiol. 202: 437-470.

Mauk, M.D. 1997. Roles of cerebellar cortex and nuclei in motor learning: Contradictions or clues? Neuron 18: 242-246.

McCrea, R.A., Strassman, A., May, E., and Highstein, S.M. 1987. Anatomical and physiological characteristics of vestibular neurons mediating the horizontal vestibulo-ocular reflex in the squirrel monkey. J. Comp. Neurol. 264: 547-570.

McElligott, J.D. and Wilson, A. 2001. Monocular vestibulo-ocular reflex (VOR) adaptation in the goldfish. Program No. 403.1. http://sfn.scholarone.com

McElligott, J.G. and Spencer, R. 2000. Neuropharmacological aspects of the vestibuloocular reflex. In Neurochemistry of the vestibular system (eds. A.J. Beitz and J.H. Anderson). CRC Press, Boca Raton, FL.

McElligott, J.G., Beeton, P., and Polk, J. 1998. Effect of cerebellar inactivation by lidocaine microdialysis on the vestibulo-ocular reflex in goldfish. J. Neurophysiol. 79: 1286-1294.

Medina, J.F., Repa, J.C., Mauk, M.D., and LeDoux, J.E. 2002. Parallels between cerebellum- and amygdala-dependent conditioning. Nat. Rev. Neurosci. 3: 122-131.

Miles, F.A. and Eighmy, B.B. 1980. Long-term adaptive changes in primate vestibuloocular reflex. I. Behavioral observations. $J$. Neurophysiol. 43: 1406-1425.

Miles, F.A. and Lisberger, S.G. 1981. Plasticity in the vestibulo-ocular reflex: A new hypothesis. Annu. Rev. Neurosci. 4: 273-299.

Miles, F.A., Braitman, D.J., and Dow, B.M. 1980a. Long-term adaptive changes in primate vestibuloocular reflex. IV. Electrophysiological observations in flocculus of adapted monkeys. J. Neurophysiol. 43: $1477-1493$.

Miles, F.A., Fuller, J.H., Braitman, D.J., and Dow, B.M. 1980b. Long-term adaptive changes in primate vestibuloocular reflex. III. Electrophysiological observations in flocculus of normal monkeys. $J$. Neurophysiol. 43: 1437-1476.

Mustari, M.J. 1988. Response properties of dorsolateral pontine units during smooth pursuit in the rhesus macaque. J. Neurophysiol. 60: $664-686$.

Nagao, S. 1989. Behavior of floccular Purkinje cells correlated with adaptation of vestibulo-ocular reflex in pigmented rabbits. Exp. Brain Res. 77: 531-540.

Nagao, S. and Kitazawa, H. 2003. Effects of reversible shutdown of the monkey flocculus on the retention of adaptation of the horizontal vestibulo-ocular reflex. Neuroscience 118: 563-570.

Nagao, S., Kitamura, T., Nakamura, N., Hiramatsu, T. and Yamada, J. 2002. Differences of the primate flocculus and ventral paraflocculus in the mossy and climbing fiber input organization. J. Comp. Neurol. 382: $480-498$.

Nelson, A.B., Krispel, C.M., Sekimjak, C., and duLac, S. 2003. Long-lasting increases in intrinsic excitability triggered by inhibition. Neuron 40: 609-620.

Noda, H. 1986. Mossy fibres sending retinal-slip, eye, and head velocity signals to the flocculus of the monkey. J. Physiol. 379: 39-60.

Ohyama, T. and Mauk, M.D. 2001. Latent acquisition of timed responses in cerebellar cortex. J. Neurosci. 21: 682-690.

Ouardouz, M. and Sastry, B.R. 2000. Mechanisms underlying LTP of inhibitory synaptic transmission in the deep cerebellar nuclei. $J$. Neurophysiol. 84: 1414-1421.

Paige, G.D. 1983. Vestibuloocular reflex and its interactions with visual following mechanisms in the squirrel monkey. I. Response characteristics in normal animals. J. Neurophysiol. 49: 134-168.

Partsalis, A.M., Zhang, Y., and Highstein, S.M. 1995. Dorsal Y group in the squirrel monkey. II. Contribution of the cerebellar flocculus to neuronal responses in normal and adapted animals. J. Neurophysiol. 73: 632-650.

Pastor, A.M., de la Cruz, R.R., and Baker, R. 1992. Characterization and adaptive modification of the goldfish vestibuloocular reflex by sinusoidal and velocity step vestibular stimulation. J. Neurophysiol. 68: 2003-2015. 
Perrett, S.P., Ruiz, B.P., and Mauk, M.D. 1993. Cerebellar cortex lesions disrupt the learning-dependent timing of conditioned eyelid responses. J. Neurosci 13: 1708-1718.

Peterson, B.W., Baker, J.F., and Houk, J.C. 1991. A model of adaptive control of vestibuloocular reflex based on properties of cross-axis adaptation. Ann. NY Acad. Sci. 627: 319-337.

Priesol, A.J., Jones, G.E.G., Tomlinson, R.D., and Broussard, D.M. 2000. Frequency-dependent effects of glutamate antagonists on the vestibulo-ocular reflex of the cat. Brain Res. 857: 252-264.

Quinn, K.J. 1998. Classical conditioning using vestibular reflexes. J. Vest. Res. 8: $117-133$.

Raymond, J.L. and Lisberger, S.G. 1996. Behavioral analysis of signals that guide learned changes in the amplitude and dynamics of the vestibulo-ocular reflex. J. Neurosci. 16: 7791-7802.

. 1998. Neural learning rules for the vestibulo-ocular reflex. J. Neurosci. 18: 9112-9129.

Raymond, J.L., Lisberger, S.G., and Mauk, M.D. 1996. The cerebellum: A neuronal learning machine? Science 272: 1126-1131.

Robinson, D.A. 1976. Adaptive gain control of vestibuloocular reflex by the cerebellum. J. Neurophysiol. 39: 954-969.

. 1981. Control of eye movements. In Handbook of physiology-the nervous system II (eds. J.M. Brockhart and V.B. Mountcastle), pp. 1275-1320. Am. Physiol. Soc., Bethesda, MD.

Salin, P.A., Malenka, R.C., and Nicoll, R.A. 1996. Cyclic AMP mediates a presynaptic form of LTP at cerebellar parallel fiber synapses. Neuron 16: $797-803$.

Sarnat, H.B. and Netsky, M.G. 1974. Evolution of the nervous system. Oxford University Press, New York.

Schultheis, L. and Robinson, D. 1981. Directional plasticity of the vestibulo-ocular reflex in the cat. Ann. NY. Acad. Sci. 374: 504-512.

Scudder, C.A. and Fuchs, A.F. 1992a. The error signal for modification of vestibuloocular reflex gain. Ann. NY Acad. Sci. 656: 884-885. 1992b. Physiological and behavioral identification of vestibular nucleus neurons mediating the horizontal vestibuloocular reflex in trained rhesus monkeys. J. Neurophysiol. 68: 244-264.

Seidman, S.H., Paige, G.D., and Tomko, D.L. 1999. Adaptive plasticity in the naso-occipital linear vestibulo-ocular reflex. Exp. Brain Res. 125: $485-494$.

Sekirnjak, C., Vissel, B., Bollinger, J., Faulstich, M., and du Lac, S. 2003. Purkinje cell synapses target physiologically unique brainstem neurons. J. Neurosci. 23: 6392-6398.

Shadmehr, R. and Holcomb, H.H. 1997. Neural correlates of motor memory consolidation. Science 277: 821-825.

Shelhamer, M., Tiliket, C., Roberts, D., Kramer, P.D., and Zee, D.S. 1994. Short-term vestibulo-ocular reflex adaptation in humans II. Error signals. Exp. Brain Res. 100: 328-336.

Shutoh, F., Katoh, A., Kitazawa, H., Aiba, A., Itohara, S., and Nagao, S. 2002. Loss of adaptability of horizontal optokinetic response eye movements in mGluR1 knockout mice. Neurosci. Res. 42: 141-145.

Squire, L.R., Slater, P.C., and Chace, P.M. 1975. Retrograde amnesia: Temporal gradient in very long term memory following electroconvulsive therapy. Science 187: 77-79.

Stahl, J.S., van Alphen, A.M., and de Zeeuw, C.I. 2000. A comparison of video and magnetic search coil recordings of mouse eye movements. J. Neurosci. Meth. 99: 101-110.

van Alphen, A.M. and de Zeeuw, C.I. 2002. Cerebellar LTD facilitated but is not essential for long-term adaptation of the vestibulo-ocular reflex. Eur. J. Neurosci. 16: 486-490.

van Alphen, A.M., Stahl, J.S., and de Zeeuw, C. 2001. The dynamic characteristics of the mouse horizontal vestibulo-ocular and optokinetic response. Brain Res. 890: 296-305.

Watanabe, E. 1984. Neuronal events corelated with long-term adaptation of the horizontal vestibulo-ocular reflex in the primate flocculus. Brain Res. 297: 169-174. 


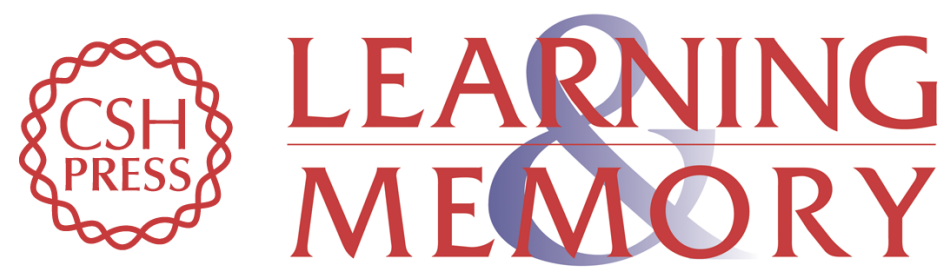

\section{Learning in a Simple Motor System}

Dianne M. Broussard and Charles D. Kassardjian

Learn. Mem. 2004, 11:

Access the most recent version at doi:10.1101//m.65804

\section{Related Content Noradrenergic Action in Prefrontal Cortex in the Late Stage of Memory Consolidation \\ Sophie Tronel, Matthijs G.P. Feenstra and Susan J. Sara \\ Learn. Mem. July , 2004 11: 453-458}

References This article cites 94 articles, 13 of which can be accessed free at: http://learnmem.cshlp.org/content/11/2/127.full.html\#ref-list-1

Articles cited in:

http://learnmem.cshlp.org/content/11/2/127.full.html\#related-urls

\section{License}

Email Alerting

Receive free email alerts when new articles cite this article - sign up in the box at the top Service right corner of the article or click here. 